\title{
Multivariate Padé Approximation for Solving Nonlinear Partial Differential Equations of Fractional Order
}

\author{
Veyis Turut $^{1}$ and Nuran Güzel ${ }^{2}$ \\ ${ }^{1}$ Department of Mathematics, Faculty of Arts and Sciences, Batman University, Batman, Turkey \\ ${ }^{2}$ Department of Mathematics, Faculty of Arts and Sciences, Yıldiz Technical University, İstanbul, Turkey \\ Correspondence should be addressed to Nuran Güzel; nguzel@yildiz.edu.tr
}

Received 27 November 2012; Revised 14 January 2013; Accepted 14 January 2013

Academic Editor: Hassan Eltayeb

Copyright (c) 2013 V. Turut and N. Güzel. This is an open access article distributed under the Creative Commons Attribution License, which permits unrestricted use, distribution, and reproduction in any medium, provided the original work is properly cited.

Two tecHniques were implemented, the Adomian decomposition method (ADM) and multivariate Padé approximation (MPA), for solving nonlinear partial differential equations of fractional order. The fractional derivatives are described in Caputo sense. First, the fractional differential equation has been solved and converted to power series by Adomian decomposition method (ADM), then power series solution of fractional differential equation was put into multivariate Padé series. Finally, numerical results were compared and presented in tables and figures.

\section{Introduction}

Recently, differential equations of fractional order have gained much interest in engineering, physics, chemistry, and other sciences. It can be said that the fractional derivative has drawn much attention due to its wide application in engineering physics [1-9]. Some approximations and numerical techniques have been used to provide an analytical approximation to linear and nonlinear differential equations and fractional differential equations. Among them, the variational iteration method, homotopy perturbation method [10, 11], and the Adomian decomposition method are relatively new approaches $[5-9,12,13]$.

The decomposition method has been used to obtain approximate solutions of a large class of linear or nonlinear differential equations $[12,13]$. Recently, the application of the method is extended for fractional differential equations [6$9,14]$.

Many definitions and theorems have been developed for multivariate Padé approximations MPA (see [15] for a survey on multivariate Padé approximation). The multivariate Padé Approximation has been used to obtain approximate solutions of linear or nonlinear differential equations [16-19].
Recently, the application of the unvariate Padé approximation is extended for fractional differential equations [20, 21].

The objective of the present paper is to provide approximate solutions for initial value problems of nonlinear partial differential equations of fractional order by using multivariate Padé approximation.

\section{Definitions}

For the concept of fractional derivative, we will adopt Caputo's definition, which is a modification of the RiemannLiouville definition and has the advantage of dealing properly with initial value problems in which the initial conditions are given in terms of the field variables and their integer order, which is the case in most physical processes. The definitions can be seen in $[3,4,22,23]$.

\section{Decomposition Method [24]}

Consider

$$
D_{* t}^{\alpha} u(x, t)=f\left(u, u_{x}, u_{x x}\right)+g(x, t), \quad m-1<\alpha \leq m .
$$


The decomposition method requires that a nonlinear fractional differential equation (1) is expressed in terms of operator form as

$$
D_{* t}^{\alpha} u(x, t)+L u(x, t)+N u(x, t)=g(x, t), \quad x>0,
$$

where $L$ is a linear operator which might include other fractional derivatives of order less than $\alpha, N$ is a nonlinear operator which also might include other fractional derivatives of order less than $\alpha, D_{* t}^{\alpha}=\partial^{\alpha} / \partial t^{\alpha}$ is the Caputo fractional derivative of order $\alpha$, and $g(x, t)$ is the source function [24].

Applying the operator $J^{\alpha}[3,4,22,23]$, the inverse of the operator $D_{* t}^{\alpha}$, to both sides of (5) Odibat and Momani [24] obtained

$$
\begin{aligned}
u(x, t)= & \sum_{k=0}^{m-1} \frac{\partial^{k} u}{\partial t^{k}}\left(x, 0^{+}\right) \frac{t^{k}}{k !}+J^{\alpha} g(x, t) \\
& -J^{\alpha}[L u(x, t)+N u(x, t)], \\
\sum_{n=0}^{\infty} u_{n}(x, t)= & \sum_{k=0}^{m-1} \frac{\partial^{k} u}{\partial t^{k}}\left(x, 0^{+}\right) \frac{t^{k}}{k !}+J^{\alpha} g(x, t) \\
& -J^{\alpha}\left[L\left(\sum_{n=0}^{\infty} u_{n}(x, t)\right)+\sum_{n=0}^{\infty} A_{n}\right] .
\end{aligned}
$$

From this, the iterates are determined in [24] by the following recursive way:

$$
\begin{aligned}
& u_{0}(x, t)=\sum_{k=0}^{m-1} \frac{\partial^{k} u}{\partial t^{k}}\left(x, 0^{+}\right) \frac{t^{k}}{k !}+J^{\alpha} g(x, t), \\
& u_{1}(x, t)=-J^{\alpha}\left(L u_{0}+A_{0}\right), \\
& u_{2}(x, t)=-J^{\alpha}\left(L u_{1}+A_{1}\right), \\
& \vdots \\
& u_{n+1}(x, t)=-J^{\alpha}\left(L u_{n}+A_{n}\right) .
\end{aligned}
$$

\section{Multivariate Padé Aproximation [25]}

Consider the bivariate function $f(x, y)$ with Taylor series development

$$
f(x, y)=\sum_{i, j=0}^{\infty} c_{i j} x^{i} y^{j}
$$

around the origin. We know that a solution of unvariate Padé approximation problem for

$$
f(x)=\sum_{i=0}^{\infty} c_{i} x^{i}
$$

is given by

$$
\begin{aligned}
& p(x)=\left|\begin{array}{cccc}
\sum_{i=0}^{m} c_{i} x^{i} & x \sum_{i=0}^{m-1} c_{i} x^{i} & \ldots & x^{n} \sum_{i=0}^{m-n} c_{i} x^{i} \\
c_{m+1} & c_{m} & \ldots & c_{m+1-n} \\
\vdots & \vdots & \ddots & \vdots \\
c_{m+n} & c_{m+n-1} & \ldots & c_{m}
\end{array}\right|, \\
& q(x)=\left|\begin{array}{cccc}
1 & x & \ldots & x^{n} \\
c_{m+1} & c_{m} & \ldots & c_{m+1-n} \\
\vdots & \vdots & \ddots & \vdots \\
c_{m+n} & c_{m+n-1} & \ldots & c_{m}
\end{array}\right|
\end{aligned}
$$

Let us now multiply $j$ th row in $p(x)$ and $q(x)$ by $x^{j+m-1}(j=$ $2, \ldots, n+1)$ and afterwards divide $j$ th column in $p(x)$ and $q(x)$ by $x^{j-1}(j=2, \ldots, n+1)$. This results in a multiplication of numerator and denominator by $x^{m n}$. Having done so, we get

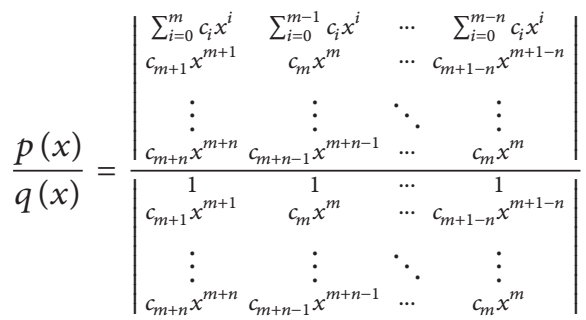

if $\left(D=\operatorname{det} D_{m, n} \neq 0\right)$.

This quotent of determinants can also immediately be written down for a bivariate function $f(x, y)$. The sum $\sum_{i=0}^{k} c_{i} x^{i}$ shall be replaced by $k$ th partial sum of the Taylor series development of $f(x, y)$ and the expression $c_{k} x^{k}$ by an expression that contains all the terms of degree $k$ in $f(x, y)$. Here a bivariate term $c_{i j} x^{i} y^{j}$ is said to be of degree $i+j$. If we define

$$
\begin{aligned}
& p(x, y) \\
& =\left|\begin{array}{cccc}
\sum_{i+j=0}^{m} c_{i j} x^{i} y^{j} & \sum_{i+j=0}^{m-1} c_{i j} x^{i} y^{j} & \ldots & \sum_{i+j=0}^{m-n} c_{i j} x^{i} y^{j} \\
\sum_{i+j=m+1} c_{i j} x^{i} y^{j} & \sum_{i+j=m} c_{i j} x^{i} y^{j} & \ldots & \sum_{i+j=m+1-n} c_{i j} x^{i} y^{j} \\
\vdots & \vdots & \ddots & \vdots \\
\sum_{i+j=m+n} c_{i j} x^{i} y^{j} & \sum_{i+j=m+n-1} c_{i j} x^{i} y^{j} & \ldots & \sum_{i+j=m} c_{i j} x^{i} y^{j}
\end{array}\right|,
\end{aligned}
$$




$$
\begin{aligned}
& q(x, y) \\
& =\left|\begin{array}{cccc}
1 & 1 & \ldots & 1 \\
\sum_{i+j=m+1} c_{i j} x^{i} y^{j} & \sum_{i+j=m} c_{i j} x^{i} y^{j} & \ldots & \sum_{i+j=m+1-n} c_{i j} x^{i} y^{j} \\
\vdots & \vdots & \ddots & \vdots \\
\sum_{i+j=m+n} c_{i j} x^{i} y^{j} & \sum_{i+j=m+n-1} c_{i j} x^{i} y^{j} & \ldots & \sum_{i+j=m} c_{i j} x^{i} y^{j}
\end{array}\right| .
\end{aligned}
$$

Then it is easy to see that $p(x, y)$ and $q(x, y)$ are of the form

$$
\begin{aligned}
& p(x, y)=\sum_{i+j=m n}^{m n+m} a_{i j} x^{i} y^{j}, \\
& q(x, y)=\sum_{i+j=m n}^{m n+n} b_{i j} x^{i} y^{j} .
\end{aligned}
$$

We know that $p(x, y)$ and $q(x, y)$ are called Padé equations [25]. So the multivariate Padé approximant of order $(m, n)$ for $f(x, y)$ is defined as

$$
r_{m, n}(x, y)=\frac{p(x, y)}{q(x, y)}
$$

\section{Numerical Experiments}

In this section, two methods, ADM and MPA, shall be illustrated by two examples. All the results are calculated by using the software Maple12. The full ADM solutions of examples can be seen from Odibat and Momani [24].

Example 1. Consider the nonlinear time-fractional advection partial differential equation [24]

$$
\begin{array}{r}
D_{* t}^{\alpha} u(x, t)+u(x, t) u_{x}(x, t)=x+x t^{2}, \\
t>0, x \in R, 0<\alpha \leq 1,
\end{array}
$$

subject to the initial condition

$$
u(x, 0)=0 .
$$

Odibat and Momani [24] solved the problem using the decomposition method, and they obtained the following recurrence relation [24]:

$$
\begin{aligned}
& u_{0}(x, t)=u(x, 0)+J^{\alpha}\left(x+x t^{2}\right) \\
&=x\left(\frac{t^{\alpha}}{\Gamma(\alpha+1)}+\frac{2 t^{\alpha+2}}{\Gamma(\alpha+3)}\right), \\
& u_{j+1}(x, t)=-J^{\alpha}\left(A_{j}\right), \quad j \geq 0,
\end{aligned}
$$

where $A_{j}$ are the Adomian polynomials for the nonlinear function $N=u u_{x}$. In view of (15), the first few components of the decomposition series are derived in [24] as follows:

$$
\begin{gathered}
u_{0}(x, t)=x\left(\frac{t^{\alpha}}{\Gamma(\alpha+1)}+\frac{2 t^{\alpha+2}}{\Gamma(\alpha+3)}\right) \\
u_{1}(x, t)=-x\left(\frac{\Gamma(2 \alpha+1) t^{3 \alpha}}{\Gamma(\alpha+1)^{2} \Gamma(3 \alpha+1)}\right. \\
+\frac{4 \Gamma(2 \alpha+3) t^{3 \alpha+2}}{\Gamma(\alpha+1) \Gamma(\alpha+3) \Gamma(3 \alpha+3)} \\
\left.+\frac{4 \Gamma(2 \alpha+5) t^{3 \alpha+4}}{\Gamma(\alpha+3)^{2} \Gamma(3 \alpha+5)}\right) \\
u_{2}(x, t)=2 x\left(\frac{\Gamma(2 \alpha+1) \Gamma(4 \alpha+1) t^{5 \alpha}}{\Gamma(\alpha+1)^{3} \Gamma(3 \alpha+1) \Gamma(5 \alpha+1)}\right. \\
\left.+\frac{8 \Gamma(2 \alpha+5) \Gamma(4 \alpha+7) t^{5 \alpha+6}}{\Gamma(\alpha+1)^{3} \Gamma(3 \alpha+5) \Gamma(5 \alpha+7)}+\cdots\right)
\end{gathered}
$$

and so on; in this manner, the rest of components of the decomposition series can be obtained [24]. by [24]

The first three terms of the decomposition series are given

$$
\begin{gathered}
u(x, t) \\
=x\left(\frac{t^{\alpha}}{\Gamma(\alpha+1)}+\frac{2 t^{\alpha+2}}{\Gamma(\alpha+3)}-\frac{\Gamma(2 \alpha+1) t^{3 \alpha}}{\Gamma(\alpha+1)^{2} \Gamma(3 \alpha+1)}\right. \\
\left.-\frac{4 \Gamma(2 \alpha+3) t^{3 \alpha+2}}{\Gamma(\alpha+1) \Gamma(\alpha+3) \Gamma(3 \alpha+3)}+\cdots\right) .
\end{gathered}
$$

For $\alpha=1(16)$ is

$$
u(x, t)=x t+0.1 \times 10^{-9} t^{3}-0.1333333333 x t^{5} .
$$

Now, let us calculate the approximate solution of (18) for $m=$ 4 and $n=2$ by using Multivariate Padé approximation. To obtain multivariate Padé equations of (18) for $m=4$ and $n=$ 2 , we use (10). By using (10), we obtain

$$
\begin{aligned}
p(x, t) & \\
= & \left|\begin{array}{ccc}
x t+0.1 \times 10^{-9} t^{3} & x t & x t \\
0 & 0.1 \times 10^{-9} t^{3} & 0 \\
-0.1333333333 x t^{5} & 0 & 0.1 \times 10^{-9} t^{3}
\end{array}\right| \\
= & 0.1333333333 \times 10^{-10} \\
& \times\left(t^{2}+0.7500000002 \times 10^{-9}\right) x^{3} t^{7},
\end{aligned}
$$




$$
\begin{aligned}
& q(x, t) \\
& =\left|\begin{array}{ccc}
1 & 1 & 1 \\
0 & 0.1 \times 10^{-9} t^{3} & 0 \\
-0.1333333333 x t^{5} & 0 & 0.1 \times 10^{-9} t^{3}
\end{array}\right| \\
& =0.1333333333 \times 10^{-10} \\
& \quad \times\left(t^{2}+0.7500000002 \times 10^{-9}\right) x^{2} t^{6} .
\end{aligned}
$$

So, the multivariate Padé approximation of order $(4,2)$ for (17), that is,

$$
\begin{aligned}
& {[4,2]_{(x, t)}=\frac{\left(t^{2}+0.7500000002 \times 10^{-9}\right) x t}{t^{2}+0.7500000002 \times 10^{-9}} .} \\
& p(x, a) \\
& =\mid \begin{array}{c}
1.128379167 x a-0.9577979850 x a^{3}+0.6018022226 x a \\
0 \\
-0.7005608116 x a^{7}
\end{array} \\
& {[6,2]_{(x, t)}=-\left(1.201464294 t^{2}-1.231832347 t\right.} \\
& -0.8326662354) \times \sqrt{t} \\
& \times(0.7379312378+1.718058483 t)^{-1} .
\end{aligned}
$$$$
=-0.4907854507\left(1.201464294 a^{4}-1.231832347 a^{2}-0.8326662354\right) x^{3} a^{11},
$$$$
q(x, a)=\left|\begin{array}{ccc}
1 & 1 & 1 \\
0 & 0.6018022226 x a^{5} & 0 \\
-0.7005608116 x a^{7} & 0 & 0.6018022226 x a^{5}
\end{array}\right|=0.4907854507\left(0.7379312378+1.718058483 a^{2}\right) x^{3} a^{10}
$$

For $\alpha=0.5(17)$ is

$$
\begin{aligned}
u(x, t)= & 1.128379167 x t^{0.5}-0.9577979850 x t^{1.5} \\
& +0.6018022226 x t^{2.5}-0.7005608116 x t^{3.5}
\end{aligned}
$$

For simplicity, let $t^{1 / 2}=a$; then

$$
\begin{aligned}
u(x, a)= & 1.128379167 x a-0.9577979850 x a^{3} \\
& +0.6018022226 x a^{5}-0.7005608116 x a^{7} .
\end{aligned}
$$

Using (10) to calculate the multivariate Padé equations for (22) we get
For $\alpha=0.75(17)$ is

$$
\begin{aligned}
u(x, t)= & 0.00007125345441 x t^{7.5} \\
& +0.1764791440 \times 10^{-5} x t^{9.5} \\
& -0.1238343301 \times 10^{-17} x t^{22.5} \\
& -0.2897967272 \times 10^{-19} x t^{24.5}
\end{aligned}
$$

For simplicity, let $t^{1 / 2}=a$; then

$$
\begin{aligned}
u(x, a)= & 0.00007125345441 x a^{15} \\
& +0.1764791440 \times 10^{-5} x a^{19} \\
& -0.1238343301 \times 10^{-17} x a^{45} \\
& -0.2897967272 \times 10^{-19} x a^{49}
\end{aligned}
$$

Using (10) to calculate the multivariate Padé equations and then recalling that $t^{1 / 2}=a$, we get multivariate Padé approximation of order $(49,2)$ for $(25)$, that is,

$$
\begin{aligned}
{[49,2]_{(x, t)}=- } & 0.8398214310 \times 10^{-39} x^{3} t^{113 / 2} \\
\times & (-0.00007125345441-0.1764791440 \\
& \left.\times 10^{-5} t^{2}+0.1238343301 \times 10^{-17} t^{15}\right) \\
& \times\left(0.8398214310 \times 10^{-39} x^{2} t^{49}\right)^{-1}
\end{aligned}
$$

Table 1, Figures 1(a), 1(b), 1(c), 2(a), 2(b), 2(c), and 2(d) shows the approximate solutions for (13) obtained for different values of $\alpha$ using the decomposition method (ADM) and the multivariate Padé approximation (MPA). The value of $\alpha=1$ is for the exact solution $u(x, t)=x t$ [24].

Example 2. Consider the nonlinear time-fractional hyperbolic equation [24]

$$
\begin{aligned}
D_{* t}^{\alpha} u(x, t)= & \frac{\partial}{\partial x}\left(u(x, t) \frac{\partial u(x, t)}{\partial x}\right), \\
& t>0, x \in R, 1<\alpha \leq 2,
\end{aligned}
$$

subject to the initial condition

$$
u(x, 0)=x^{2}, \quad u_{t}(x, 0)=-2 x^{2} .
$$




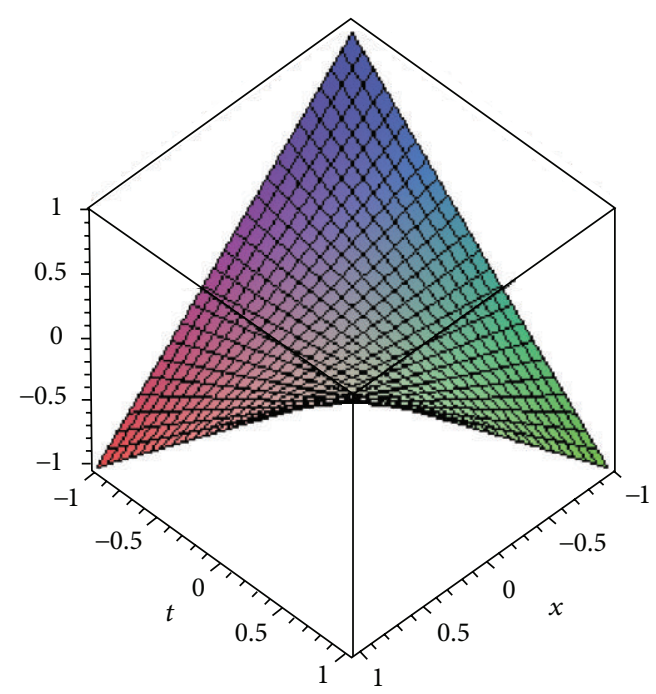

(a)

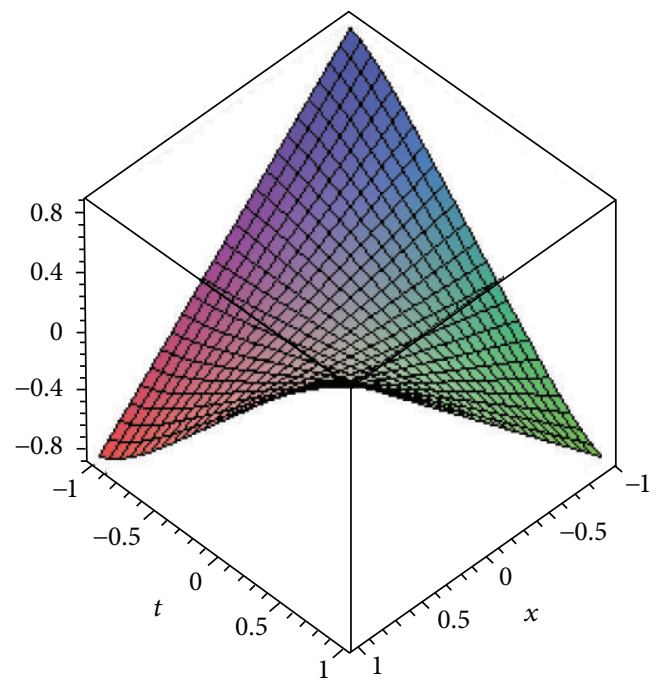

(b)

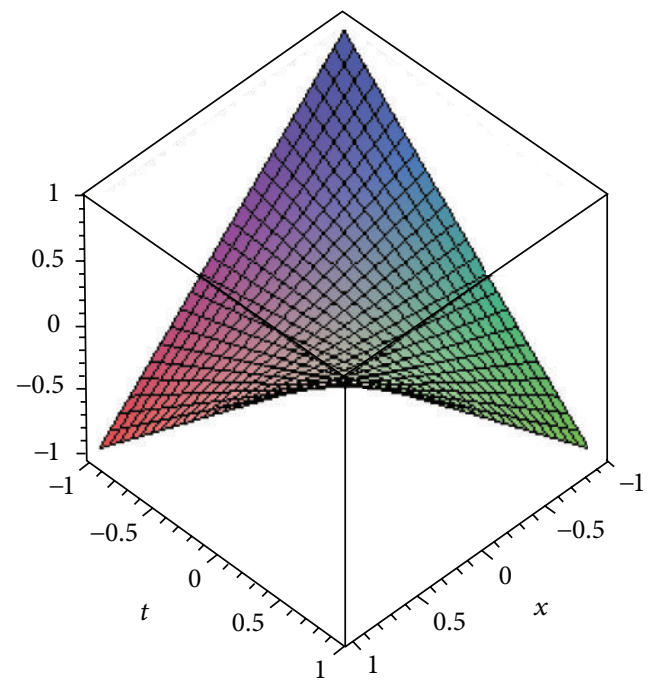

(c)

FIGURE 1: (a) Exact solution of Example 1 for $\alpha=1$ (b) ADM solution of Example 1 for $\alpha=1$ (c) Multivariate Padé approximation of ADM solution for $\alpha=1$ in Example 1 .

Odibat and Momani [24] solved the problem using the decomposition method, and they obtained the following recurrence relation in [24]:

$$
\begin{gathered}
u_{0}(x, t)=u(x, 0)+t u_{x}(x, 0)=x^{2}(1-2 t), \\
u_{j+1}(x, t)=J^{\alpha}\left(A_{j}\right)_{x}, \quad j \geq 0
\end{gathered}
$$

where $A_{j}$ are the Adomian polynomials for the nonlinear function $N=u u_{x}$. In view of (30), the first few components of the decomposition series are derived in [24] as follows:

$$
\begin{aligned}
& u_{1}(x, t)= 6 x^{2}\left(\frac{t^{\alpha}}{\Gamma(\alpha+1)}-\frac{4 t^{\alpha+1}}{\Gamma(\alpha+2)}+\frac{8 t^{\alpha+2}}{\Gamma(\alpha+3)}\right) \\
& u_{2}(x, t)= 72 x^{2}\left(\frac{t^{2 \alpha}}{\Gamma(2 \alpha+1)}-\frac{4 t^{2 \alpha+1}}{\Gamma(2 \alpha+2)}\right. \\
&\left.\quad+\frac{8 t^{2 \alpha+2}}{\Gamma(2 \alpha+3)}-\frac{2 \Gamma(\alpha+2) t^{2 \alpha+1}}{\Gamma(\alpha+1) \Gamma(2 \alpha+2)}\right) \\
&+72 x^{2}\left(\frac{8 \Gamma(\alpha+3) t^{2 \alpha+2}}{\Gamma(\alpha+2) \Gamma(2 \alpha+3)}\right. \\
&\left.-\frac{16 \Gamma(\alpha+4) t^{2 \alpha+3}}{\Gamma(\alpha+3) \Gamma(2 \alpha+4)}\right)
\end{aligned}
$$

$u_{0}(x, t)=x^{2}(1-2 t)$ 


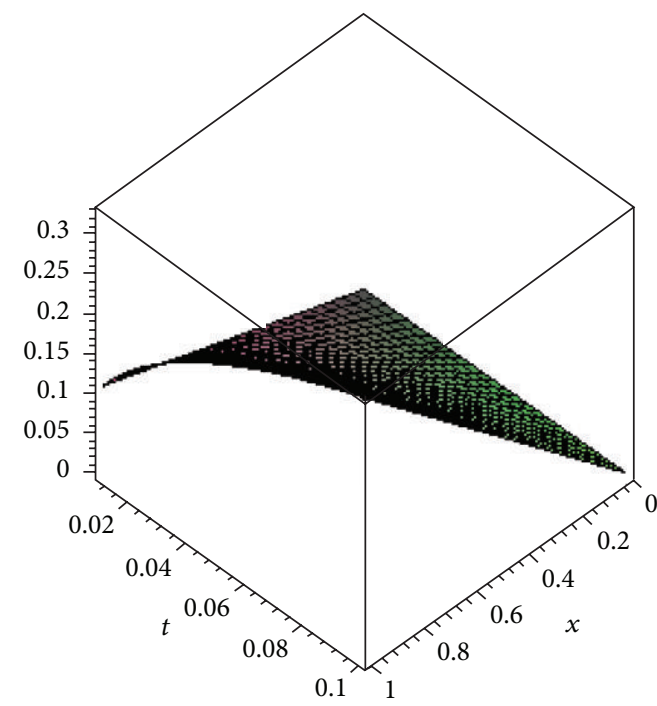

(a)

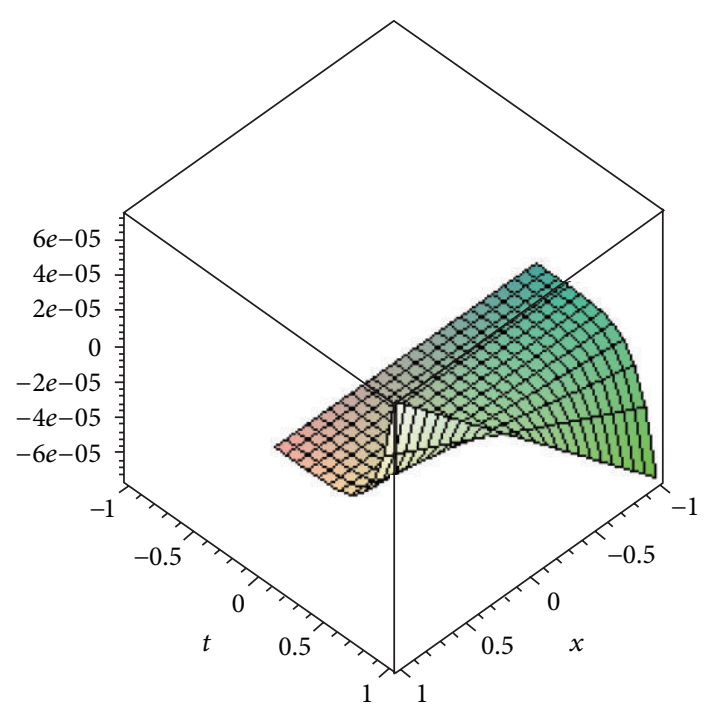

(c)

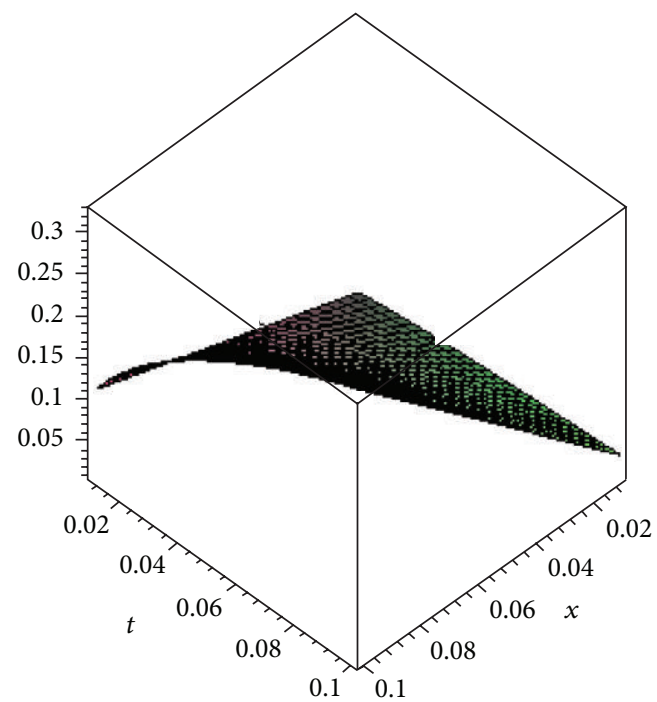

(b)

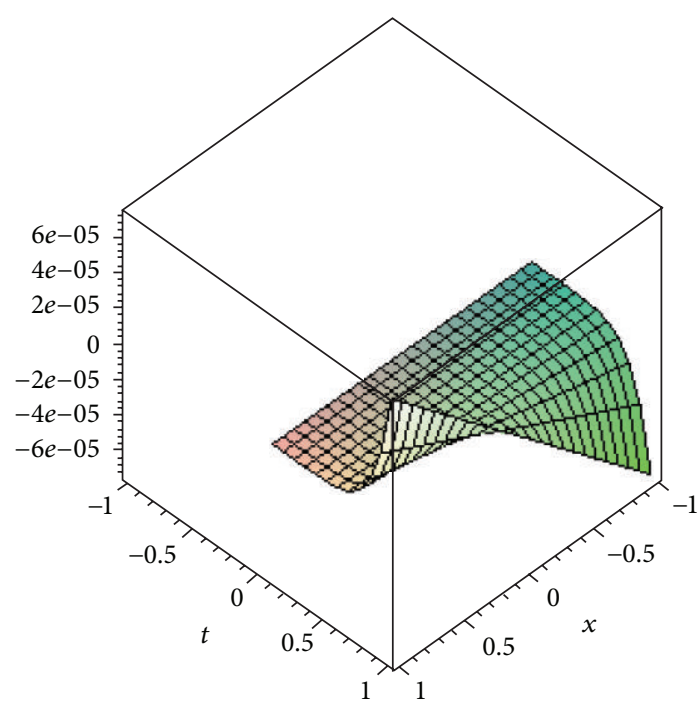

(d)

Figure 2: (a) ADM solution of Example 1 for $\alpha=0.5$ (b) Multivariate Padé approximation of ADM solution for $\alpha=0.5$ in Example 1 (c) ADM solution of Example 1 for $\alpha=0.75$ (d) Multivariate Padé approximation of ADM solution for $\alpha=0.75$ in Example 1 .

and so on; in this manner the rest of components of the decomposition series can be obtained.

The first three terms of the decomposition series (7) are given [24] by

$$
\begin{aligned}
u(x, t)= & x^{2}(1-2 t)+6 x^{2} \\
& \times\left(\frac{t^{\alpha}}{\Gamma(\alpha+1)}-\frac{4 t^{\alpha+1}}{\Gamma(\alpha+2)}+\frac{8 t^{\alpha+2}}{\Gamma(\alpha+3)}\right) \\
& +72 x^{2}\left(\frac{t^{2 \alpha}}{\Gamma(2 \alpha+1)}+\cdots\right) .
\end{aligned}
$$

For $\alpha=2(43)$ is

$$
\begin{aligned}
u(x, t)= & x^{2}(1-2 t) \\
& +6 x^{2}\left(0.5000000000 t^{2}-0.6666666668 t^{3}\right. \\
& \left.+0.3333333334 t^{4}\right) \\
& +3.000000000 x^{2} t^{4} .
\end{aligned}
$$

Now, let us calculate the approximate solution of (33) for $m=$ 4 and $n=2$ by using multivariate Padé approximation. To 


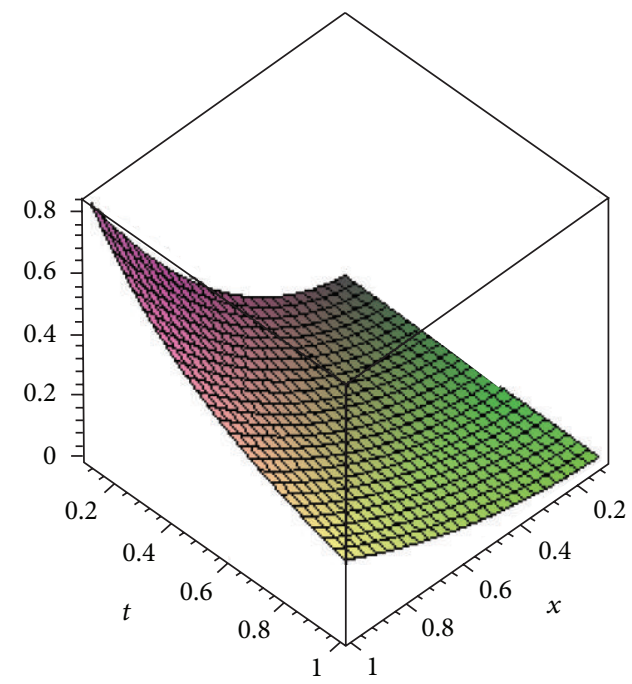

(a)

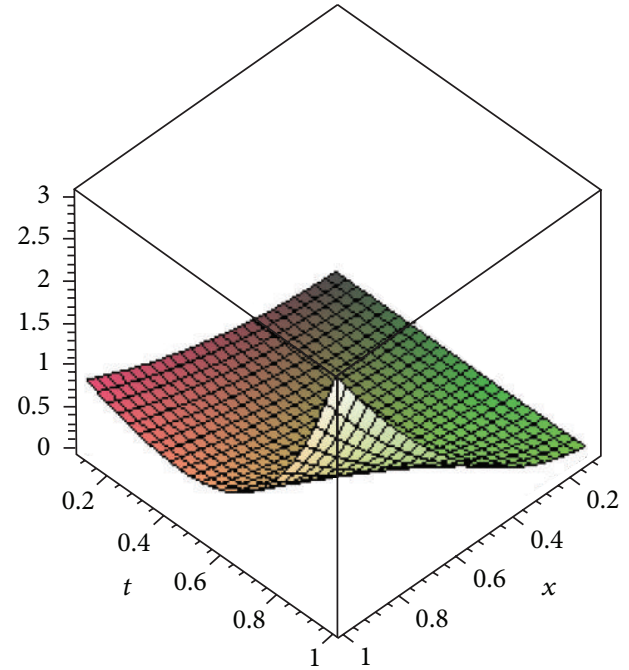

(b)

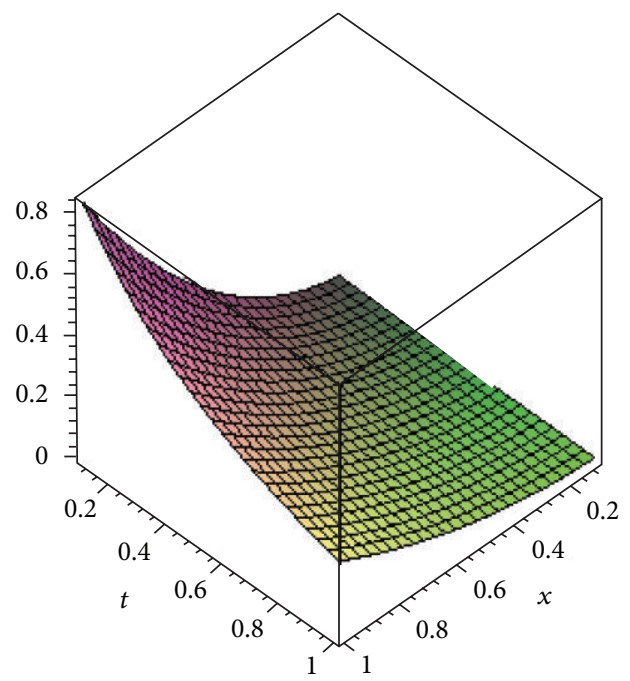

(c)

Figure 3: (a) Exact solution of Example 2 for $\alpha=2.0$ (b) ADM solution of Example 2 for $\alpha=2.0$ (c) Multivariate Padé approximation of ADM solution for $\alpha=2.0$ in Example 2 .

obtain multivariate Padé equations of (33) for $m=4$ and $n=$ 2 , we use (10). By using (10), we obtain

$$
\begin{aligned}
& p(x, t) \\
& =\left|\begin{array}{ccc}
x^{2}(1-2 t)+3.000000000 x^{2} t^{2} & x^{2}(1-2 t) & x^{2} \\
-4.000000001 x^{2} t^{3} & 3.000000000 x^{2} t^{2} & -2 x^{2} t \\
5.000000000 x^{2} t^{4} & -4.000000001 x^{2} t^{3} & 3.000000000 x^{2} t^{2}
\end{array}\right| \\
& =-20.00000000 t^{4}\left(0.28 \times 10^{-9} t^{2}-0.34 \times 10^{-9} t(-0.04999999986) x^{6},\right. \\
& q(x, t) \\
& =\left|\begin{array}{ccc}
1 & 1 & 1 \\
-4.000000001 x^{2} t^{3} & 3.000000000 x^{2} t^{2} & -2 x^{2} t \\
5.000000000 x^{2} t^{4} & -4.000000001 x^{2} t^{3} & 3.0000000000 x^{2} t^{2}
\end{array}\right| \\
& =20.00000000 t^{4}\left(0.04999999999+0.1000000001 t+0.0500000004 t^{2}\right) x^{4} .
\end{aligned}
$$

So, the multivariate Padé approximation of order $(4,2)$ for (33), that is,

$$
\begin{gathered}
{[4,2]_{(x, t)}=-1.000000000\left(0.28 \times 10^{-9} t^{2}-0.34 \times 10^{-9} t\right.} \\
-0.04999999986) x^{2} \\
\times(0.0499999999+0.1000000001 t \\
\left.+0.0500000004 t^{2}\right)^{-1} .
\end{gathered}
$$



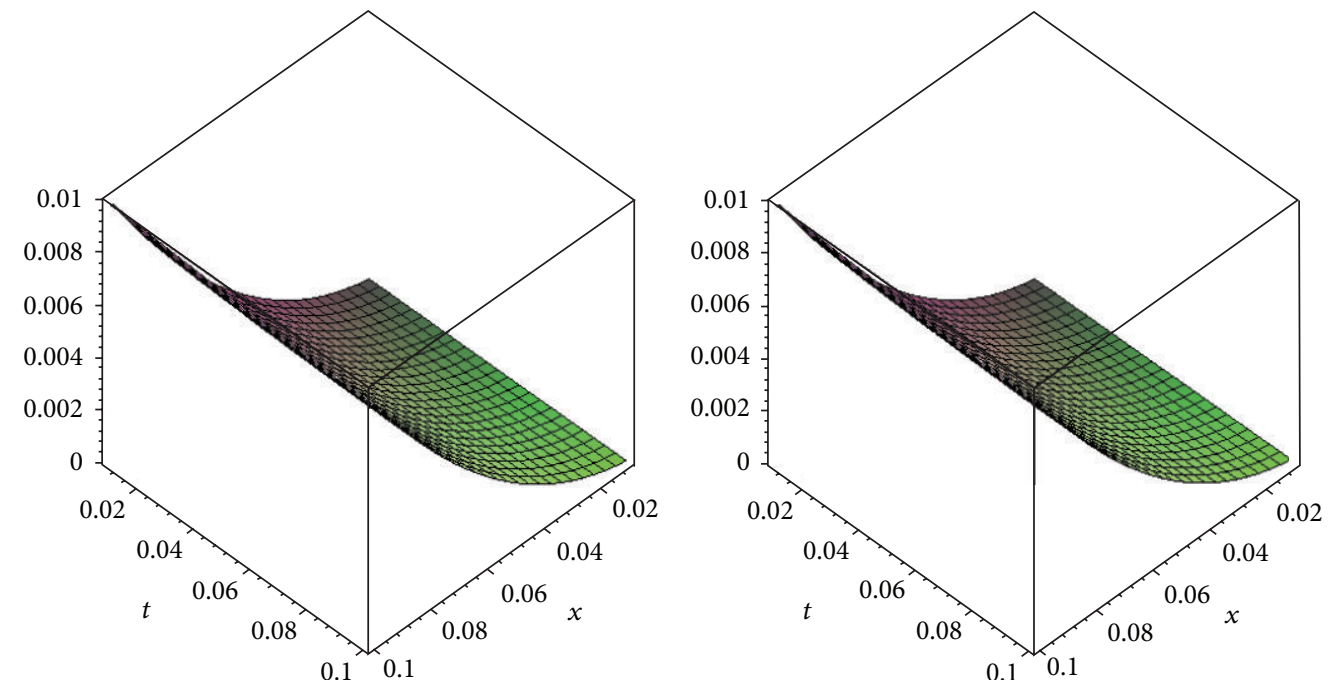

(a)

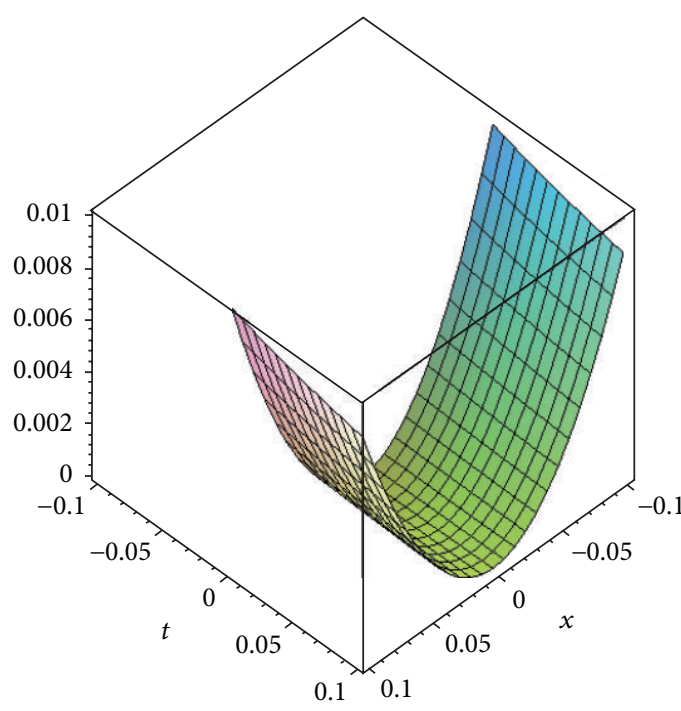

(c)

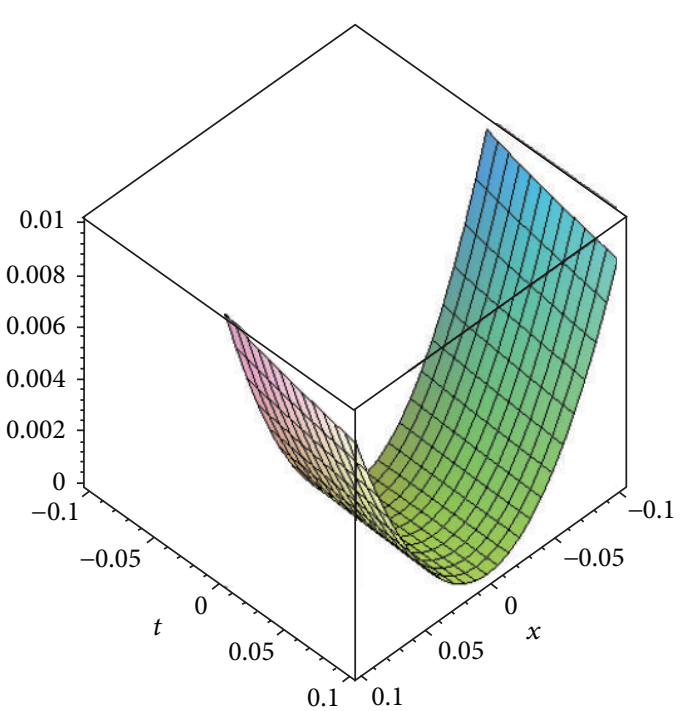

(d)

Figure 4: (a) ADM solution of Example 2 for $\alpha=1.5$ (b) Multivariate Padé approximation of ADM solution for $\alpha=1.5$ in Example 2 (c) ADM solution of Example 2 for $\alpha=1.75$ (d) Multivariate Padé approximation of ADM solution for $\alpha=1.75$ in Example 2.

For $\alpha=1.5(32)$ is

$$
\begin{aligned}
u(x, t)= & x^{2}(1-2 t)+6 x^{2} \\
& \times\left(0.7522527782 t^{1.5}-1.203604445 t^{2.5}\right. \\
& \left.\quad+0.6877739683 t^{3.5}\right)+12.00000000 x^{2} t^{3.0}
\end{aligned}
$$

For simplicity, let $t^{1 / 2}=a$; then

$$
u(x, a)=x^{2}\left(1-2 a^{2}\right)+6 x^{2}
$$

$$
\begin{aligned}
& \times\left(0.7522527782 a^{3}\right. \\
& \left.-1.203604445 a^{5}+0.6877739683 t^{7}\right) \\
+ & 12.00000000 x^{2} a^{6} . \\
= & x^{2}-x^{2} 2 a^{2}+4.513516669 x^{2} a^{3} \\
- & 7.221626670 x^{2} a^{5}+4.126643810 x^{2} a^{7} \\
+ & 12.00000000 x^{2} a^{6} .
\end{aligned}
$$

Using (10) to calculate multivariate Padé equations of (37) for $m=7$ and $n=2$, we use (10). By using (10), we obtain 


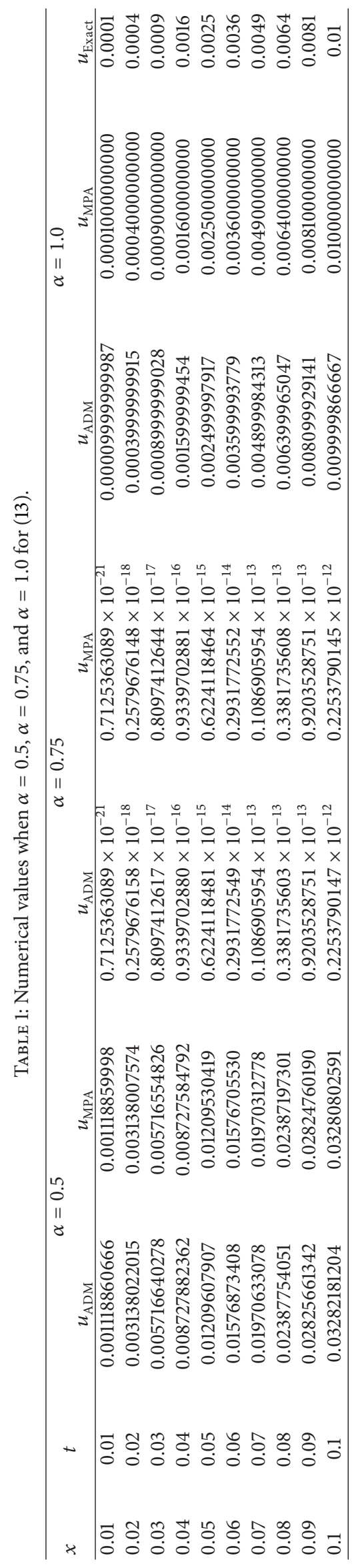




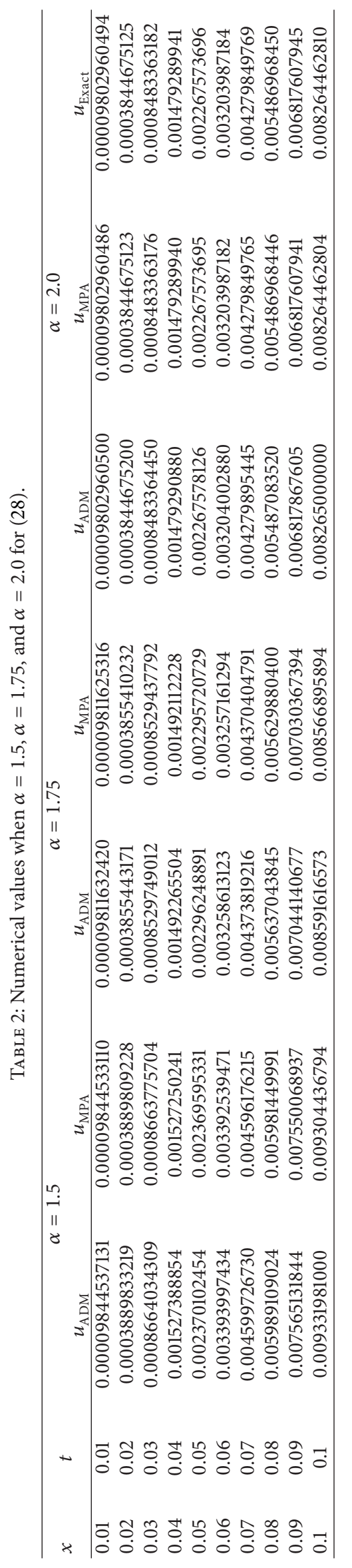




$$
\begin{aligned}
& p(x, a)=\left|\begin{array}{ccc}
x^{2}-x^{2} 2 a^{2}+4.513516669 x^{2} a^{3}-7.221626670 x^{2} a^{5} & x^{2}-x^{2} 2 a^{2}+4.513516669 x^{2} a^{3} & x^{2}-x^{2} 2 a^{2}+4.513516669 x^{2} a^{3} \\
12.00000000 x^{2} t^{6} & -7.221626670 x^{2} a^{5} & 0 \\
4.126643810 x^{2} a^{7} & 12.00000000 x^{2} a^{6} & -7.221626670 x^{2} a
\end{array}\right| \\
& =49.51972572\left(8.235760151 a^{5}+0.879185531 a^{4}+1.253427636 a^{3}+1.403426542 a^{2}+1.750000001 a+1.053153890\right) x^{6} a^{10}, \\
& q(x, a)=\left|\begin{array}{ccc}
1 & 1 & 1 \\
12.00000000 x^{2} a^{6} & -7.221626670 x^{2} a^{5} & 0 \\
4.126643810 x^{2} a^{7} & 12.00000000 x^{2} a^{6} & -7.221626670 x^{2} a
\end{array}\right| \\
& =49.51972572\left(1.053153890+1.750000001 a+3.509734322 a^{2}\right) x^{4} a^{10},
\end{aligned}
$$

recalling that $t^{1 / 2}=a$, we get multivariate Padé approximation of order $(7,2)$ for $(36)$, that is,

$$
\begin{aligned}
{[7,2]_{(x, t)}=(} & 8.235760151 t^{5 / 2}+0.879185531 t^{2} \\
& +1.253427636 t^{3 / 2}+1.403426542 t \\
& +1.750000001 \sqrt{t}+1.053153890) x^{2} \\
& \times(1.053153890+1.750000001 \sqrt{t} \\
& +3.509734322 t)^{-1}
\end{aligned}
$$

For $\alpha=1.75(32)$ is

$$
\begin{aligned}
u(x, t)= & x^{2}(1-2 t) \\
& +6 x^{2}\left(0.6217515726 t^{1.75}-0.9043659240 t^{2.75}\right. \\
& \left.+0.4823284927 t^{3.75}\right) \\
& +6.189965715 x^{2} t^{3.5}
\end{aligned}
$$

For simplicity, let $t^{1 / 4}=a$; then

$$
\begin{aligned}
u(x, a)= & x^{2}\left(1-2 a^{4}\right) \\
& +6 x^{2}\left(0.6217515726 a^{7}-0.9043659240 a^{11}\right. \\
& \left.+0.4823284927 a^{15}\right)+6.189965715 x^{2} a^{14} \\
= & x^{2}-2 x^{2} a^{4}+3.730509436 x^{2} a^{7} \\
& -5.426195544 x^{2} a^{11}+2.893970956 x^{2} a^{15} \\
& +6.189965715 x^{2} a^{14} .
\end{aligned}
$$

Using (10) to calculate multivariate Padé equations of (41) for $m=15$ and $n=2$, we use (10). By using (10), we obtain

$$
\begin{aligned}
& p(x, a) \\
& =\left|\begin{array}{ccc}
x^{2}-2 x^{2} a^{4}+3.730509436 x^{2} a^{7}-5.426195544 x^{2} a^{11} & x^{2}-2 x^{2} a^{4}+3.730509436 x^{2} a^{7}-5.426195544 x^{2} a^{11} & x^{2}-2 x^{2} a^{4}+3.730509436 x^{2} a^{7}-5.426195544 x^{2} a^{11} \\
6.189965715 x^{2} a^{14} & 0 & 0 \\
2.893970956 x^{2} a^{15} & 6.189965715 x^{2} a^{14} & 0
\end{array}\right| \\
& =-38.31567556 x^{6} a^{28}\left(-1+2 a^{4}-3.730509436 a^{7}+5.426195544 a^{11}\right), \\
& q(x, a)=\left|\begin{array}{ccc}
1 & 1 & 1 \\
6.189965715 x^{2} a^{14} & 0 & 0 \\
2.893970956 x^{2} a^{15} & 6.189965715 x^{2} a^{14} & 0
\end{array}\right|=38.31567556 x^{4} a^{28}
\end{aligned}
$$

recalling that $t^{1 / 4}=a$, we get multivariate Padé approximation of order $(15,2)$ for $(40)$, that is,

$$
\begin{aligned}
{[15,2]_{(x, t)}=} & -38.31567556 x^{6} t^{7} \\
\times & \left(-1+2 t-3.730509436 t^{7 / 4}\right. \\
& \left.+5.426195544 t^{11 / 4}\right) \\
& \times\left(38.31567556 x^{4} t^{7}\right)^{-1}
\end{aligned}
$$

Table 2, Figures 3(a), 3(b), 3(c), 4(a), 4(b), 4(c), and 4(d) show the approximate solutions for (28) obtained for different values of $\alpha$ using the decomposition method (ADM) and the multivariate Padé approximation (MPA). The value of $\alpha=2$ is for the exact solution $u(x, t)=(x / t+1)^{2}$ [24].

\section{Concluding Remarks}

The fundamental goal of this paper has been to construct an approximate solution of nonlinear partial differential 
equations of fractional order by using multivariate Padé approximation. The goal has been achieved by using the multivariate Padé approximation and comparing with the Adomian decomposition method. The present work shows the validity and great potential of the multivariate Padé approximation for solving nonlinear partial differential equations of fractional order from the numerical results. Numerical results obtained using the multivariate Padé approximation and the Adomian decomposition method are in agreement with exact solutions.

\section{References}

[1] J. H. He, "Nonlinear oscillation with fractional derivative and its applications," in Proceedings of International Conference on Vibrating Engineering, pp. 288-291, Dalian, China, 1998.

[2] J. H. He, "Some applications of nonlinear fractional differential equations and their approximations," Bulletin of Science and Technology, vol. 15, no. 2, pp. 86-90, 1999.

[3] Y. Luchko and R. Gorenflo, The Initial Value Problem for Some Fractional Differential Equations with the Caputo Derivative, Series A08-98, Fachbreich Mathematik und Informatik, Freic Universitat Berlin, Berlin, Germany, 1998.

[4] I. Podlubny, Fractional Differential Equations, vol. 198 of Mathematics in Science and Engineering, Academic Press, San Diego, Calif, USA, 1999.

[5] S. Momani, "Non-perturbative analytical solutions of the spaceand time-fractional Burgers equations," Chaos, Solitons \& Fractals, vol. 28, no. 4, pp. 930-937, 2006.

[6] Z. M. Odibat and S. Momani, "Application of variational iteration method to nonlinear differential equations of fractional order," International Journal of Nonlinear Sciences and Numerical Simulation, vol. 7, no. 1, pp. 27-34, 2006.

[7] S. Momani and Z. Odibat, "Analytical solution of a timefractional Navier-Stokes equation by Adomian decomposition method," Applied Mathematics and Computation, vol. 177, no. 2, pp. 488-494, 2006.

[8] S. Momani and Z. Odibat, "Numerical comparison of methods for solving linear differential equations of fractional order," Chaos, Solitons \& Fractals, vol. 31, no. 5, pp. 1248-1255, 2007.

[9] Z. M. Odibat and S. Momani, "Approximate solutions for boundary value problems of time-fractional wave equation," Applied Mathematics and Computation, vol. 181, no. 1, pp. 767774, 2006.

[10] G. Domairry and N. Nadim, "Assessment of homotopy analysis method and homotopy perturbation method in non-linear heat transfer equation," International Communications in Heat and Mass Transfer, vol. 35, no. 1, pp. 93-102, 2008.

[11] G. Domairry, M. Ahangari, and M. Jamshidi, "Exact and analytical solution for nonlinear dispersive $K(m, p)$ equations using homotopy perturbation method," Physics Letters A, vol. 368, no. 3-4, pp. 266-270, 2007.

[12] G. Adomian, "A review of the decomposition method in applied mathematics," Journal of Mathematical Analysis and Applications, vol. 135, no. 2, pp. 501-544, 1988.

[13] G. Adomian, Solving Frontier Problems of Physics: The Decomposition Method, vol. 60 of Fundamental Theories of Physics, Kluwer Academic Publishers, Dordrecht, The Netherlands, 1994.
[14] S. Momani, "An explicit and numerical solutions of the fractional KdV equation," Mathematics and Computers in Simulation, vol. 70, no. 2, pp. 110-118, 2005.

[15] Ph. Guillaume and A. Huard, "Multivariate Padé approximation," Journal of Computational and Applied Mathematics, vol. 121, no. 1-2, pp. 197-219, 2000.

[16] V. Turut, E. Çelik, and M. Yiğider, "Multivariate Padé approximation for solving partial differential equations (PDE)," International Journal for Numerical Methods in Fluids, vol. 66, no. 9, pp. 1159-1173, 2011.

[17] V. Turut and N. Güzel, "Comparing numerical methods for solving time-fractional reaction-diffusion equations," ISRN Mathematical Analysis, vol. 2012, Article ID 737206, 28 pages, 2012.

[18] V. Turut, "Application of Multivariate padé approximation for partial differential equations," Batman University Journal of Life Sciences, vol. 2, no. 1, pp. 17-28, 2012.

[19] V. Turut and N. Güzel, "On solving partial differential eqauations of fractional order by using the variational iteration method and multivariate padé approximation," European Journal of Pure and Applied Mathematics. Accepted.

[20] S. Momani and R. Qaralleh, "Numerical approximations and Padé approximants for a fractional population growth model," Applied Mathematical Modelling, vol. 31, no. 9, pp. 1907-1914, 2007.

[21] S. Momani and N. Shawagfeh, "Decomposition method for solving fractional Riccati differential equations," Applied Mathematics and Computation, vol. 182, no. 2, pp. 1083-1092, 2006.

[22] K. B. Oldham and J. Spanier, The Fractional Calculus, Academic Press, New York, NY, USA, 1974.

[23] M. Caputo, "Linear models of dissipation whose Q is almost frequency independent. Part II," Journal of the Royal Astronomical Society, vol. 13, no. 5, pp. 529-539, 1967.

[24] Z. Odibat and S. Momani, "Numerical methods for nonlinear partial differential equations of fractional order," Applied Mathematical Modelling, vol. 32, no. 1, pp. 28-39, 2008.

[25] A. Cuyt and L. Wuytack, Nonlinear Methods in Numerical Analysis, vol. 136 of North-Holland Mathematics Studies, NorthHolland Publishing, Amsterdam, The Netherlands, 1987. 


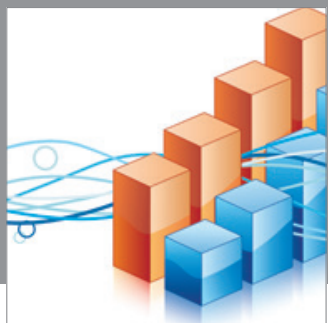

Advances in

Operations Research

mansans

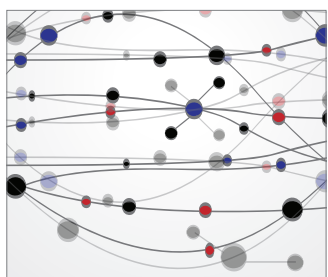

The Scientific World Journal
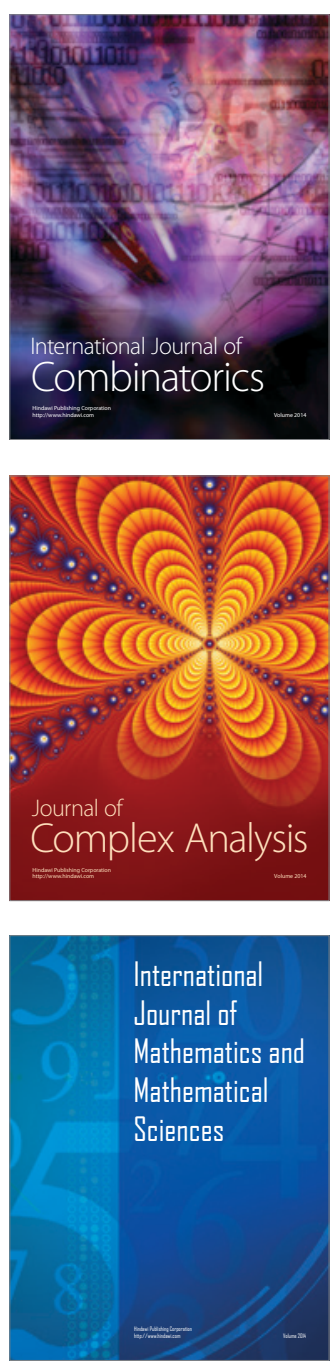
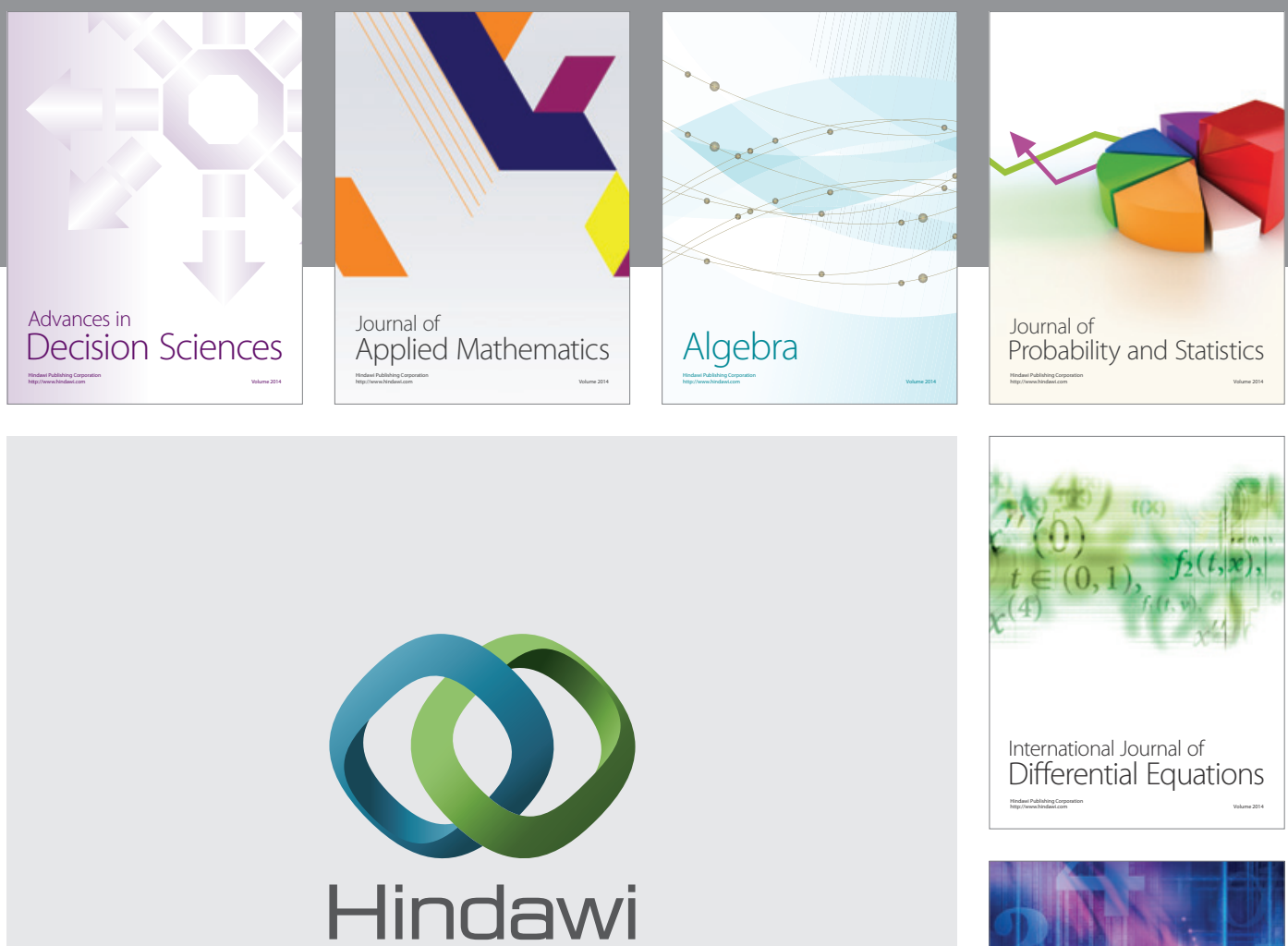

Submit your manuscripts at http://www.hindawi.com
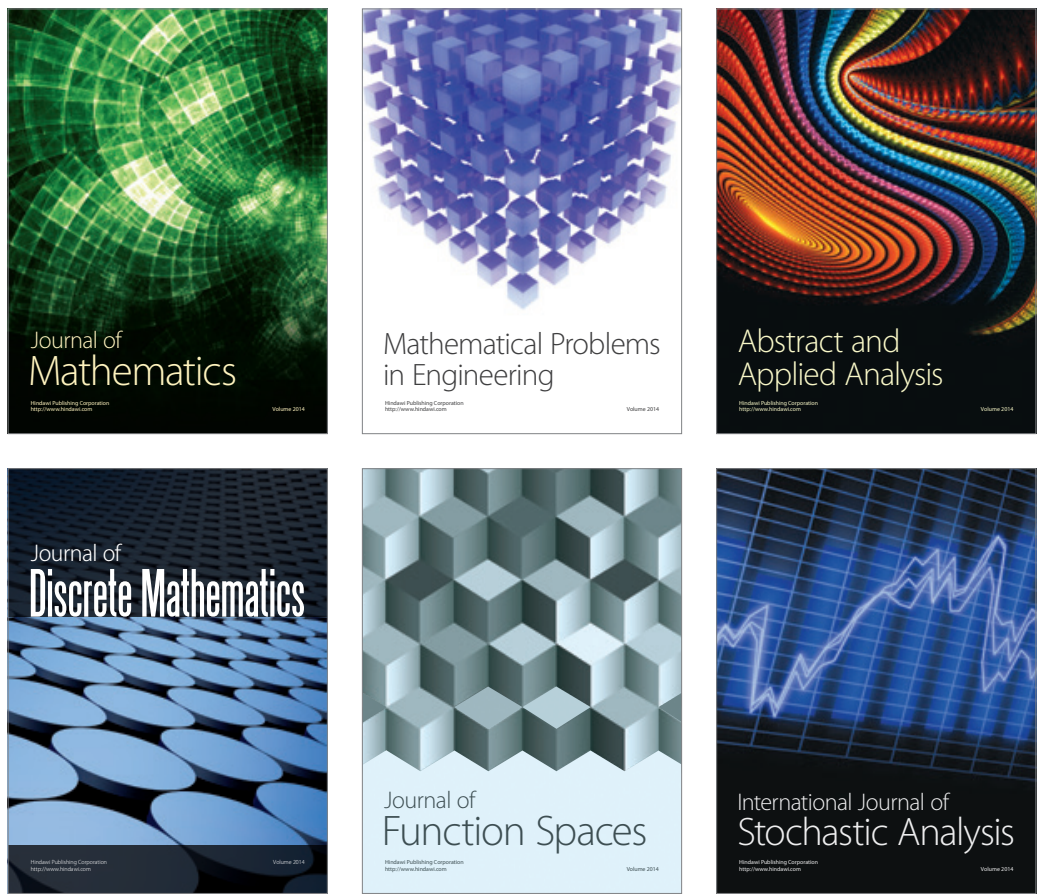

Journal of

Function Spaces

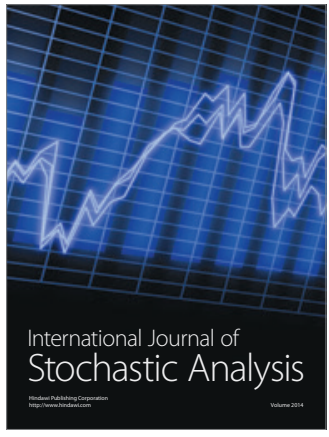

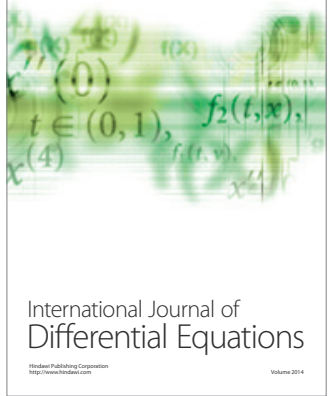
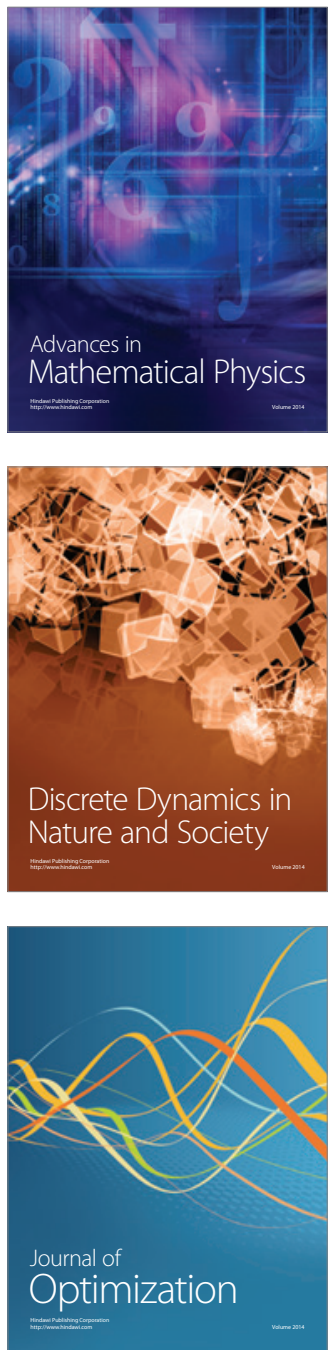